\title{
-NOTES-
}

\section{THE POST BUCKLING BEHAVIOR OF A CLAMPED CIRCULAR PLATE*}

By SOL R. BODNER (Polytechnic Institute of Brooklyn)

1. Introduction. The von Kármán equations for large deflections of elastic plates have been completely solved by K. O. Friedrichs and J. J. Stoker for the case of a circular plate simply supported around the circumference where it is subjected to a uniform radial thrust in the plane of the plate, Refs. [1] and [2]. Their solution describes the plate behavior from the initial buckled state to the condition of the ratio of the edge thrust to initial buckling load becoming infinitely large.

The purpose of this note is to apply the methods developed in [1] to determine the post buckling behavior of a clamped circular plate. A modification of one of the methods is made which reduces the computation necessary to analyze certain aspects of the plate behavior.

2. Mathematical formulation. For assumed radially symmetric deformations, the von Kármán equations for a circular plate are a pair of non-linear ordinary differential equations, each of second order. These equations are

$$
\begin{gathered}
\nabla^{4} \phi=w_{, r r}(1 / r) w_{, r}, \\
(\gamma h)^{2} \nabla^{4} w+(1 / r) \phi_{, r} w_{, r r}+(1 / r) \phi_{, r r} w_{, r}=0
\end{gathered}
$$

where $\phi$ is the stress function of the membrane stresses, $w$ is the deflection of the middle surface of the plate, $r$ is the radial coordinate from the plate center, $h$ is the plate thickness and $\gamma^{2}=1 / 12\left(1-\nu^{2}\right)$ where $\nu$ is Poisson's ratio. The radius of the plate is $R$ and the quantities obtained by differentiating $\phi$ are stresses per modulus of elasticity, $E$. Equations (1) and (2) can be simplified by introducing new dependent variables $p$ and $q$ where

$$
\begin{gathered}
p=(1 / r) \phi_{, r}, \text { the compressive radial membrane } \\
\text { stress divided by Young's modulus, } \\
q=-(R / r) w_{, r} .
\end{gathered}
$$

Equations (1) and (2) then become

$$
\begin{gathered}
\left\{r^{2}\left[G p-(1 / 2) q^{2}\right]\right\}_{, r}=0 \\
\left\{r^{2}\left[\eta^{2} G q+p q\right]\right\}_{. r}=0
\end{gathered}
$$

where

$$
G q \equiv\left(r^{3} q_{, r}\right)_{,}\left(R^{2} / r^{3}\right)
$$

and

$$
\eta^{2}=\gamma^{2} h^{2} / R^{2}
$$

*Received Dec. 9, 1953. 
The integration of (5) and (6) yields

$$
\begin{gathered}
G p-(1 / 2) q^{2}=0, \\
\eta^{2} G q+p q=0 .
\end{gathered}
$$

The constants arising from the integration are zero by continuity considerations. Equations (9) and (10) with appropriate boundary conditions completely define the problem. The boundary conditions at the edge of a clamped plate subjected to a radial edge thrust $p^{*}$ are

$$
\begin{array}{lll}
p=p^{*} & \text { at } & r=R, \\
q=0 & \text { at } & r=R .
\end{array}
$$

The two other boundary conditions needed for a complete solution are at the center of the plate and are due to symmetry requirements. They are

$$
\begin{array}{lll}
p_{. r}=0 & \text { at } & r=0, \\
q_{. r}=0 & \text { at } & r=0 .
\end{array}
$$

3. Methods of solution. The boundary value problem considered here depends essentially upon one parameter $N$ : the ratio of the edge pressure $p^{*}$ to the lowest critical pressure $p_{\text {or }}$. In [1] and [2] a perturbation method is used to solve (9) and (10) for low values of $N, 1 \leq N \leq 2.5$. This perturbation method consists of expanding $p, q$ and $p^{*}$ as power series in a quantity $\epsilon$ which vanishes at the onset of buckling, i.e. at $N=1$. The value of $p_{\text {cr }}$ is obtained as one of the steps in the perturbation method and was found to be

$$
p_{\text {cr }}=14.68 \eta^{2} .
$$

This method becomes increasingly tedious as $N$ increases and it is convenient to use a power series method for $N>2.5$.

The power series method is mathematically simpler than the perturbation method but necessitates an estimate of the membrane stress and the curvature at the center of the plate. This estimate can be obtained from the results of the application of the perturbation method in the region of lower $N$. The power series method can be applied, however, without previous use of the perturbation method by utilizing an important aspect of the plate behavior.

One of the notable results of [1] and [2] was that at large values of $N$ the membrane stresses become tensions in the interior of the simply supported plate and change abruptly to compressions in a narrow "boundary layer" at the plate edge. Since the same general behavior can be expected to occur for a clamped plate, the power series method can be modified to obtain the value of $N$ at which the radial membrane stress at the center of the plate, $p_{0}$, changes from compression to tension. The solution of this problem can serve to give the estimate of the physical quantities needed for the further direct application of the power series method.

An asymptotic solution is used to determine the limit situation as $N$ becomes infinitely large. From this solution the limiting value of the membrane stress in the interior of the plate can be determined.

4. Power series method. The introduction of a new independent variable $\alpha$ and new dependent variables $\pi$ and $\kappa$ into (9) and (10) permits a solution of those equations by power series expansions. 
Hence, if

$$
\left.\begin{array}{ll}
\alpha=A r / R, & 0 \leq \alpha \leq A, \\
\pi=p / A^{2} \eta^{2}, & \kappa=q / A^{2} \eta
\end{array}\right\}
$$

where $A$ is an arbitrary parameter, (9) and (10) become

$$
\begin{aligned}
& \left(1 / \alpha^{3}\right)\left(\alpha^{3} \pi_{, \alpha}\right)_{, \alpha}=(1 / 2) \kappa^{2}, \\
& \left(1 / \alpha^{3}\right)\left(\alpha^{3} \kappa_{, \alpha}\right)_{, \alpha}+\pi \kappa=0,
\end{aligned}
$$

with boundary conditions

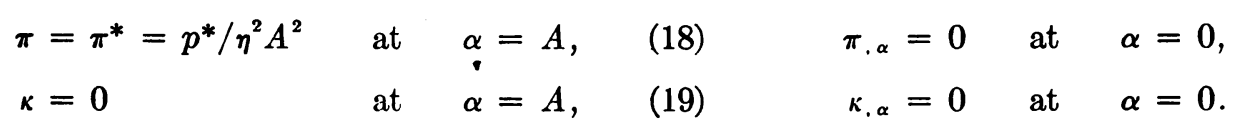

The variables $\pi$ and $\kappa$ can be represented in the form

$$
\begin{aligned}
& \pi=\sum_{k=0}^{\infty} \pi_{k} \alpha^{2 k}, \\
& \kappa=\sum_{k=0}^{\infty} \kappa_{k} \alpha^{2 k} .
\end{aligned}
$$

The first term in each of these expansions is a function of physical quantities at the center of the plate. The remaining coefficients of each series can be expressed as functions of the first terms, $\pi_{0}$ and $\kappa_{0}$, by means of the recursion relations obtained from substituting (22) and (23) into the differential equations (16) and (17). These recursion relations are

$$
\begin{aligned}
2 k(2 k+2) \pi_{k} & =(1 / 2) \sum_{m+n=k-1} \kappa_{m} \kappa_{n}, \\
2 k(2 k+2) \kappa_{k} & =-\sum_{m+n=k-1} \pi_{m} \kappa_{n} .
\end{aligned}
$$

The power series method of [1] and [2] consists of prescribing $\pi_{0}$ and $\kappa_{0}$ and solving for $A$ and $\pi^{*}$ from the equations obtained from boundary conditions (19) and (18). The corresponding values of $p, q, p^{*}$ and $N$ can then be calculated from the previous formulas.

The value of $A$ is the lowest root of the equation

$$
\sum_{k=0}^{\infty} \kappa_{k} A^{2 k}=0,
$$

which is boundary condition (19) at the plate edge. This equation can be solved for $A$ with a minimum of labor if $A \sim 1$. For $A=1, \pi_{0}=p_{0} / \eta^{2}$ and $\kappa_{0}=q_{0} / \eta$ where $p_{0}$ and $q_{0}$ are the values of $p$ and $q$ at the center of the plate. These values can be estimated for a particular $N$ from the results obtained by the application of the perturbation method for lower values of $N$.

From the results of [1] and [2] it is reasonable to expect that, for the case of a clamped plate, an $N$ exists for which $p_{0}=0$. Therefore, for this special case, $\pi_{0}=0$ and all the coefficients in the expansions (22) and (23) can be written in terms of one parameter, $\kappa_{0}$. The value of $\kappa_{0}$ can be determined from the solution of (26) in which $A$ can be prescribed 
to be unity. That is, $\kappa_{0}$ is the lowest root of

$$
\sum_{k=0}^{\infty} \kappa_{k}=0,
$$

which was found to be $\kappa_{0}=27.11$ using coefficients up to $\kappa_{20}$. The corresponding value of $\pi^{*}$ was found from (22) and (18) to be 29.11. The value of $N$ for the condition $p_{0}=0$ was found from the relations $N=p^{*} / p_{\mathrm{cr}}=\pi^{*} / 14.68$ to be 1.98 .

With this information the first section of the curve for the variation of the radial membrane stress at the plate center, $p_{0}$, with $N$ can be sketched as shown in Fig. 1.

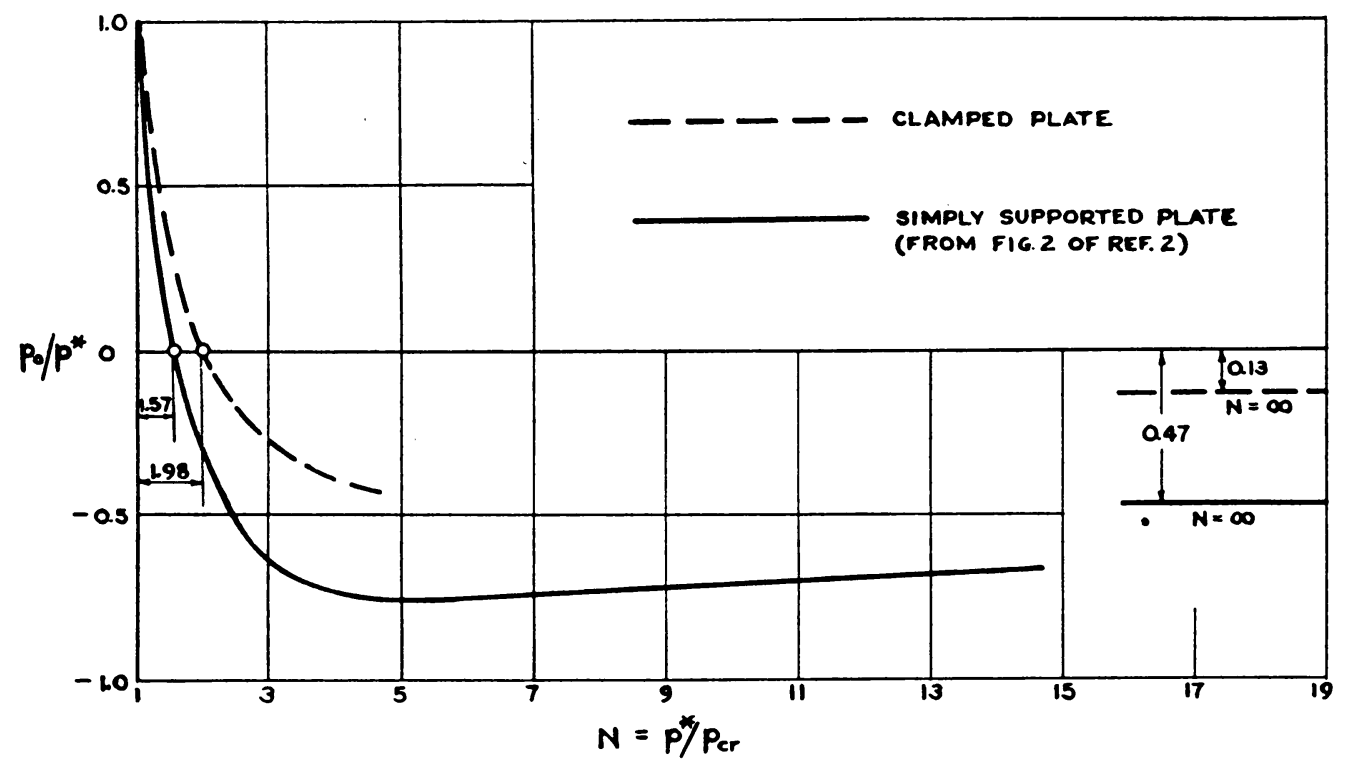

FIG. 1. Radial membrane stress at center of plate.

A similar curve could be drawn for $q_{0}$. The general behavior of these curves, from physical considerations, should not differ from those obtained in [2] for simply supported plates. These curves can then serve to provide estimates of $p_{0}$ and $q_{0}$ for use in the further application of the power series method. When the limiting case of $N \rightarrow \infty$ is solved, an estimate of $p_{0}$ and $q_{0}$ can be obtained for the complete range of $N$, (Fig. 1). In this manner the power series method can be conveniently used for the range $1 \leq N \leq 15$, thereby being independent of the results of the perturbation method. For $N$ about equal to and greater than 15 , the power series method becomes unwieldly and an asymptotic development is preferable.

5. Asymptotic solution. The solution of (9) and (10) for $N$ tending to infinity for the clamped plate follows, in general, the method described in [1] for the simply supported plate. The computation necessary for the clamped plate was greater than that required for the simply supported plate. This solution shows that as $N \rightarrow \infty$ the interior of the plate acts as a membrane subjected to a uniform tensile stress equal to .131 times the compressive thrust at the plate edge. That is,

$$
\lim _{N \rightarrow \infty} p=-.131 p^{*} \text {. }
$$

This is also the asymptotic value of $p_{0}$ and is indicated on the graph, (Fig. 1). 


\section{ACKNOWLEDGMLET}

This paper stems from a master's thesis submitted to New York University. The author wishes to express his thanks to Professor K. O. Friedrichs, his thesis advisor, for his advice and criticism.

\section{REFERENCES}

1. K. O. Friedrichs and J. J. Stoker, The non-linear boundary value problem of the buckled plate, Am. J. Math., 63, 839-888 (1941).

2. K. O. Friedrichs and J. J. Stoker, Buckling of the circular plate beyond the critical thrust, J. Appl. Mech., 9, 7-14 (1942).

\section{AN APPROXIMATE SOLUTION TO THE NAVIER-STOKES EQUATIONS*}

BY MORTON MITCHNER (Harvard L'niversity)

The purpose of this note is to show how a new approximate solution of the NavierStokes equations may be constructed from any given exact solution having a certain specified form. We shall suppose that we are given an exact solution of the Navier-Stokes equations for an incompressible viscous fluid having a velocity field $\mathbf{q}^{\prime}=\left(q_{1}^{\prime}, q_{2}^{\prime}, q_{3}^{\prime}\right)=$ $\left(u^{\prime}, v^{\prime}, w^{\prime}\right)$ specified in the form

$$
\begin{aligned}
u^{\prime} & =\alpha U(y, z, t), \\
v^{\prime} & =\alpha V(y, z, t), \\
w^{\prime} & =\alpha W(y, z, t) .
\end{aligned}
$$

$U, V$, and $W$ denote three functions of the position vector $\mathbf{r}=\left(x_{1}, x_{2}, x_{3}\right)=(x, y, z)$, and the time coordinate $t ; \alpha$ denotes a dimensionless constant. For consistency with the equations of motion (upon taking the divergence of the Navier-Stokes equation, and employing the continuity condition), the pressure $p^{\prime}$ (and density $\rho$ ) must satisfy

$$
-\frac{1}{\rho} \nabla^{2} p^{\prime}=\sum_{i, k} \frac{\partial q_{i}^{\prime}}{\partial x_{k}} \frac{\partial q_{k}^{\prime}}{\partial x_{i}}
$$

Hence, it is sufficient to assume that the pressure field has the form

$$
p^{\prime}=\alpha^{2} P(y, z, t) \text {. }
$$

Assuming the existence of the above exact solution, we can construct a new approximate solution $[q=(u, v, w), p]$ of the equations of motion for an incompressible viscous fluid, and this solution is given by

$$
\begin{aligned}
u & =u_{0}(a+b y)+u^{\prime}-\left(t-t_{0}\right) b u_{0} v^{\prime}, \\
v & =v^{\prime}, \\
w & =w^{\prime}, \\
p & =\text { constant, }
\end{aligned}
$$

\footnotetext{
${ }^{*}$ Received Dec. 26, 1952. Revised manuscript received Jan. 7, 1954.
} 